\title{
Torsional Alfvén waves: magneto-seismology in static and dynamic coronal plasmas
}

\author{
R. J. Morton, M. S. Ruderman, and R. Erdélyi \\ Solar Physics and Space Plasma Research Centre ( $\left.\mathrm{SP}^{2} \mathrm{RC}\right)$, University of Sheffield, Hicks Building, Hounsfield Road, \\ Sheffield S3 7RH, UK \\ e-mail: [r.j.morton;m.s.ruderman; robertus]@sheffield.ac.uk
}

Received 4 April 2011 / Accepted 4 August 2011

\begin{abstract}
Aims. We study the properties of torsional Alfvén waves in coronal loops so that they may be exploited for coronal seismological applications.

Methods. The governing equation is obtained for standing torsional Alfvén waves of a dynamic, gravitationally stratified plasma. The footpoints are assumed to obey line-tying conditions necessary for standing oscillations. Solutions are found in a number of different but typical scenarios to demonstrate the possibilities for both temporal and spatial magneto-seismology exploitation of waveguides with the standing torsional Alfvén oscillations.

Results. It is found that the frequency of the standing Alfvén oscillation increases as the stratification of the plasma increases. The ratio of the periods of the fundamental mode and the first overtone is also found to change as the stratification of the plasma increases. Further, the eigenfunctions of the higher overtones of the standing oscillations are found to experience a shift of their anti-nodes. The influence of a dynamic plasma on the amplitudes of the mode is also investigated. The amplitude of the torsional Alfvén mode is found to increase as the plasma within the coronal loop experiences cooling.
\end{abstract}

Key words. magnetohydrodynamics (MHD) - plasmas - Sun: corona - waves

\section{Introduction}

The ubiquitous nature of waves and oscillations resolved in magnetic structures in the solar atmosphere is beginning to be documented (Aschwanden et al. 1999; Wang et al. 2003; Tomczyk et al. 2007; Taroyan \& Erdélyi 2008; Jess et al. 2009; Morton et al. 2011). The theory of waves in magnetic flux tubes in static, homogenous plasmas is well understood (e.g., Edwin \& Roberts 1983; Erdélyi \& Fedun 2010). To date, the majority of the theoretically predicted waves have been observed in the solar corona. The first observations of waves resolved in coronal loops only reported relatively large amplitude oscillations, which were interpreted as standing fast kink modes (e.g., Aschwanden et al. 1999). Propagating and standing periodic intensity oscillations have also been observed and interpreted as slow magnetoacoustic modes (for the latest reviews see Banerjee et al. 2007; De Moortel 2009; and Taroyan \& Erdélyi 2009). These events appeared to be relatively infrequent considering the continuous sources of driving available in the lower solar atmosphere, e.g. $p$-modes, granular buffeting (see, e.g., Fedun et al. 2011a, for simulations of both scenarios), vortex motions (observed by Bonet et al. 2008; and simulations by Fedun et al. 2011b). Tomczyk et al. (2007) and Erdélyi \& Taroyan (2008) also reported small amplitude propagating fast kink modes in coronal loops that appear to be ubiquitous and ever present. How these continuous waves, perhaps generated in the lower solar atmosphere, reach the corona is still a matter of debate, although some guiding by the magnetic field lines appears likely (Fedun et al. 2011a,b).
The only predicted mode from the theory of oscillations in a cylindrical waveguide with a straight magnetic field (e.g., Edwin \& Roberts 1983) that has not yet been observed in the corona is the (torsional) Alfvén wave. This is likely due to the fact that the mode is highly incompressible, so it does not cause a (yet observable) modulation of the intensity. The suggested method for the detection of the Alfvén mode is the use of spectral line broadening (Erdélyi et al. 1998; Zaqarashvili 2003). However, out of phase broadening on opposite edges of a coronal loop is required to confirm their presence. Unfortunately the resolution onboard current satellites is of the order of the loops radius, hence it is too coarse for the observation of the mode. The Alfvén wave has been observed though in the lower solar atmosphere in a magnetic bright point (Jess et al. 2009) with remarkable confirmation of a number of theoretically predicted properties (i.e., radial dependence, phase relation).

The prospect of magneto-seismology with Alfvén waves was first highlighted by Zaqarashvili \& Murawski (2007) for coronal applications and Verth et al. (2010) for the lower solar atmosphere. The properties of the torsional Alfvén wave, i.e. frequency, amplitude, are only dependent upon the internal parameters of the waveguide, whereas the fast kink mode is also dependent upon the external plasma parameters. Magnetoseismology with Alfvén waves requires less input parameters, e.g. plasma density, hence the relative error of parameters required for diagnostics will be less. It follows that any errors on estimates of plasma quantities, e.g., magnetic field strength, scale height, from the seismology should be reduced. There are a number of proposed sources of Alfvén waves, e.g. vortex 
motions in the lower solar atmosphere (Fedun et al. 2011b; Shelyag et al. 2011) which can drive torsional motions; resonant absorption (Goossens et al. 1992; Erdelyi \& Goossens 1995; Erdélyi et al. 1995; Erdelyi 1998) or damping of fast kink oscillations (Ruderman \& Roberts 2002; Goossens et al. 2002; Terradas et al. 2010) in the corona which cause quasi-Alfvén waves (although not the torsional mode), hence their presence is expected to be ubiquitous in the corona.

We can gain some insight into which properties of the waveguide will have significant influence by referring to the literature on magneto-seismology of fast kink oscillations (see Andries et al. 2009; and Ruderman \& Erdélyi 2009, for reviews). It has been found that magnetic structuring (Verth \& Erdélyi 2008; Ruderman et al. 2008) and density structuring (Andries et al. 2005; Dymova \& Ruderman 2006a; Erdélyi \& Verth 2007; Verth et al. 2007) along the loop axis have the dominant influence on kink modes in static loops. Other waveguide properties have a much smaller (but nonetheless important) influence, e.g. loop shape (Dymova \& Ruderman 2006b; Ruderman 2009; Morton \& Erdélyi 2009a), cross-section (Ruderman 2003; Erdélyi \& Morton 2009; Morton \& Ruderman 2011), etc.

One important, but little studied, aspect that influences wave propagation is plasma dynamics. The majority of EUV coronal loops are reported to show constant downflows (see, e.g., Del Zanna 2008) indicating that they are in the radiative phase of their heating/cooling cycle (see, e.g., Aschwanden \& Terradas 2008; Aschwanden \& Tsiklauri 2009; Klimchuk et al. 2010, and references within). The effect this phenomenon has on fast kink oscillations has been modelled by Morton \& Erdélyi (2009b) and Ruderman (2010, 2011). It was demonstrated in Morton \& Erdélyi (2009b) that the frequency of the oscillations, the period ratio and anti-node shifts all experienced timedependent behaviour. The fast kink mode was also shown to be amplified by the mass loss (Ruderman 2011). The inclusion of background plasma dynamics has also been shown to cause additional damping of slow magneto-acoustic modes in both conductively (Erdélyi et al. 2011) and radiatively (Morton et al. 2010) cooling plasmas.

The aim of this paper is to extend the current knowledge of magneto-seismology by torsional Alfvén waves to coronal wave guides (see, e.g., Verth et al. 2010, for lower solar atmospheric waveguides). First, we investigate the effect of density stratification on the properties of standing torsional Alfvén waves, highlighting any magneto-seismological tools that should be exploited. Second, we investigate how introducing dynamics into the background plasma (in the form of radiative cooling) influences the properties of standing torsional Alfvén waves. The paper is organised as follows. In Sect. 2 we derive the governing equation for linear Alfvén waves in a stratified and dynamic plasma. Section 3 discusses how Alfvén waves propagate in a gravitationally stratified plasma. We highlight and analyse how the stratification can influence the period ratio and the spatial position of the anti-nodes. In Sect. 4 we go on to show how the inclusion of dynamic behaviour of the plasma, i.e. mass loss from the loop driven by radiative cooling, can cause amplification of the Alfvén waves.

\section{Governing equations}

We are interested in how standing torsional Alfvén waves oscillate in realistic coronal loop models. It has been shown that the influence of loop curvature on fast kink oscillations in a thin magnetic flux tube is small enough to neglect the curvature (Terradas et al. 2006; van Doorsselaere et al. 2009). We also assume the same for the following analysis but to our knowledge this remains to be shown.

Most coronal loops appear to be in a radiative cooling phase. We shall model this phenomenon. It has been observed that loops are subject to an exponential temperature decrease during this phase (Aschwanden \& Terradas 2008),

$T(t)=T_{0} \exp \left(\frac{-t}{\tau_{\text {cool }}}\right)$,

where $T_{0}$ is the initial temperature when radiative cooling begins and $\tau_{\text {cool }}$ is the characteristic radiative cooling time of the plasma.

We choose to neglect the influence of expansion of the loop cross-section as this has been studied by Verth et al. (2010) for a static equilibrium. Note that the loop expansion will affect the eigenfrequencies and eigenfunctions of the Alfvén waves significantly and may limit the applicability of our results. We assume that there is the background plasma flow directed along the loop, and all background quantities vary along the loop and with time. Then, using cylindrical coordinates $r, \varphi, z$ with the $z$-axis parallel to the loop axis, we obtain that the background state is described by the following system of equations,

$\rho\left(\frac{\partial U}{\partial t}+U \frac{\partial U}{\partial z}\right)=-\frac{\partial p}{\partial z}-\rho g \cos \theta$,

$\frac{\partial \rho}{\partial t}+\frac{\partial(U \rho)}{\partial z}=0$

$p=\frac{k_{\mathrm{B}}}{m} \rho T$.

Here Eq. (2) is the momentum equation projected on the tangent to the loop; $\rho, p, T$ and $U$ are the unperturbed density, pressure, temperature, and flow velocity, respectively; $g$ is the acceleration due to gravity, $\theta$ the angle between the tangent to the loop and the vertical direction, $k_{\mathrm{B}}$ the Boltzmann constant and $m$ the mean mass per particle.

Substituting Eqs. (1) and (4) into Eq. (2) we obtain

$\left(\frac{\partial U}{\partial t}+U \frac{\partial U}{\partial z}\right)=-g\left(\frac{H(t)}{\rho} \frac{\partial \rho}{\partial z}+\cos \theta\right)$

where

$H(t)=\frac{k_{\mathrm{B}} T(t)}{m g}$

is the atmospheric scale height. From Eq. (3) we can estimate that $U \sim L / \tau_{\text {cool }}$, which, for typical loop parameters, e.g. $L \sim$ $120 \mathrm{Mm}$ and $\tau_{\text {cool }}=1000 \mathrm{~s}$, gives flows of $U=120 \mathrm{~km} \mathrm{~s}^{-1}$. This value for the flow is at the maximum of the range of downflows observed in coronal loops (see, e.g., Schrijver et al. 1999; Ofman \& Wang 2008), where typical values range between 40$120 \mathrm{~km} \mathrm{~s}^{-1}$. The ratio of the left hand side to the right hand side of Eq. (5) for $g=274 \mathrm{~m}^{2} \mathrm{~s}^{-1}$, typical in the solar atmosphere, is of the order 1/3 for large flows, which means that the ratio is small enough to neglect the left-hand side. Assuming the loop has a semi-circular shape and is of length $2 L$ centered on $z=0$, then from Eq. (5) it follows that the projected density profile is given by

$\rho(z, t)=\rho_{\mathrm{f}} \exp \left(-\frac{h_{\mathrm{a}}}{H(t)} \cos \frac{\pi z}{2 L}\right)$. 
Here $\rho_{\mathrm{f}}$ is the density at the footpoints of the loop and $h_{\mathrm{a}}$ is the loop height above the solar atmosphere. This analysis follows that given in Ruderman (2011) and we find a similar density profile. Equation (7) is the same as that intuitively suggested by Morton \& Erdélyi (2009b) in a cooling loop. We do not derive the flow profile here as the flow is not needed in the analytic solutions. However, substitution of Eq. (7) into (3) will provide a partial differential equation for calculating an approximate flow profile. The flow generated by the cooling will have a speed proportional to $L / \tau_{\text {cool }}$, i.e., loop half length $L$ divided by the characteristic cooling timescale. For typical values in coronal loops, $L \sim 80 \mathrm{Mm}$ and $\tau_{\text {cool }} \sim 1200 \mathrm{~s}$, gives $U \sim 60 \mathrm{~km} \mathrm{~s}^{-1}$. This is inline with observed values of down-flows in coronal loops, 20-100 $\mathrm{km} \mathrm{s}^{-1}$ (e.g., Tripathi et al. 2009).

Now, consider the standing torsional Alfvén waves in the coronal loops. Recall that the only non-zero perturbed quantities in torsional Alfvén waves are the plasma displacement in the $\varphi$-direction, $\xi_{\varphi}$, and the $\varphi$-component of the magnetic field, $b_{\varphi}$. Hence, the linearized MHD equations describing the torsional Alfvén waves are

$\left(\frac{\partial}{\partial t}+U \frac{\partial}{\partial z}\right)^{2} \xi_{\varphi}=\frac{B}{\mu_{0} \rho_{i}} \frac{\partial b_{\varphi}}{\partial z}$,

$\left(\frac{\partial}{\partial t}+U \frac{\partial}{\partial z}\right) b_{\varphi}=B \frac{\partial}{\partial z}\left(\frac{\partial}{\partial t}+U \frac{\partial}{\partial z}\right) \xi_{\varphi}-b_{\varphi} \frac{\partial U}{\partial z}$

Now, expanding and rearranging Eq. (9) we arrive at

$\left(\frac{\partial}{\partial t}+U \frac{\partial}{\partial z}+\frac{\partial U}{\partial z}\right) b_{\varphi}=B\left(\frac{\partial}{\partial t}+U \frac{\partial}{\partial z}+\frac{\partial U}{\partial z}\right) \frac{\partial \xi_{\varphi}}{\partial z}$,

and canceling the derivatives from each side we arrive at

$b_{\varphi}=B \frac{\partial \xi_{\varphi}}{\partial z}$.

Substituting Eq. (11) into (8) we obtain the equation for $\xi_{\varphi}$,

$\left(\frac{\partial}{\partial t}+U \frac{\partial}{\partial z}\right)^{2} \xi_{\varphi}=v_{\mathrm{A}}^{2}(z, t) \frac{\partial^{2} \xi_{\varphi}}{\partial z^{2}}$,

where

$v_{\mathrm{A}}(z, t)=\frac{B}{\sqrt{\mu_{0} \rho(z, t)}}$,

is the Alfvén speed.

In what follows we restrict our analysis to standing oscillations, so we impose the frozen-in boundary conditions at the loop footpoints,

$\xi_{\varphi}=0 \quad$ at $\quad z= \pm L$,

where $L$ is the half-length of the loop.

\section{Alfvén waves in static plasmas}

Before we investigate the influence of dynamics on standing torsional Alfvén waves it is informative to investigate how these waves are modified by an inhomogeneous static plasma. For now we assume that the background flow, $U$, is equal to zero, hence the density is constant in time but is stratified along the loop, i.e. $\rho=\rho(z)$. Equation (12) reduces to

$\frac{\partial^{2} \xi_{\varphi}}{\partial t^{2}}-v_{\mathrm{A}}^{2}(z) \frac{\partial^{2} \xi_{\varphi}}{\partial z^{2}}=0$

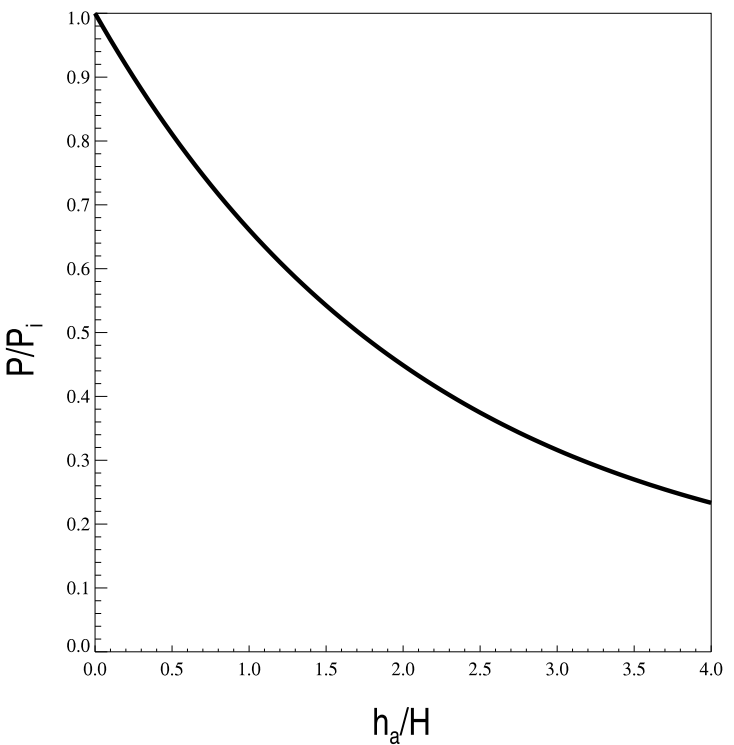

Fig. 1. Shown is the dependence of the period of the fundamental standing Alfvén mode on the loop stratification. The values of the period of the fundamental mode are normalised with respect to the value of period for a homogeneous loop $\left(P_{i}\right)$.

The Alfvén speed in this equation is now time-independent, hence we can Fourier analyse with respect to time and take $\xi_{\varphi} \propto \exp (-\mathrm{i} \omega t)$ to obtain

$\frac{\partial^{2} \xi_{\varphi}}{\partial z^{2}}+\frac{\omega^{2}}{v_{\mathrm{A}}^{2}} \xi_{\varphi}=0$,

Eq. (16) along with the boundary conditions (14) constitute a boundary-value problem known as the Sturm-Liouville problem. The eigenvalue in this problem is $\omega^{2}$. The general theory of Sturm-Liouville problems tells us that the eigenvalues of this problem are real (see, e.g., Coddington 1955). It is straightforward to show that they are also positive, so that $\omega$ is real.

For a static plasma, i.e. $U=0$, the density profile of the plasma is defined by Eq. (7) with $H=$ const., i.e. the value of scale height remains constant as the loop remains at a given temperature. Substituting the time-independent density profile into Eq. (16) gives

$\frac{\partial^{2} \xi_{\varphi}}{\partial z^{2}}+\frac{\omega^{2}}{v_{\mathrm{Af}}^{2}} \exp \left(-\frac{h_{\mathrm{a}}}{H} \cos \frac{\pi z}{2 L}\right) \xi_{\varphi}=0$,

where $\rho_{\mathrm{f}}$ and

$v_{\mathrm{Af}}=\frac{B}{\sqrt{\mu_{0} \rho_{\mathrm{f}}}}$

are the density and Alfvén speed respectively at the footpoints. We solve Eq. (17) with boundary conditions (14) numerically to obtain the eigenfrequencies. The period of the fundamental mode is plotted as a function of stratification in Fig. 1. It can be seen that, as the stratification of the plasma increases, the period of the oscillation decreases from the value obtained for a homogenous loop.

\subsection{Temporal seismology}

A standard tool that has been developed for magneto-seismology with, e.g. fast kink, sausage and longitudinal oscillations, is the 


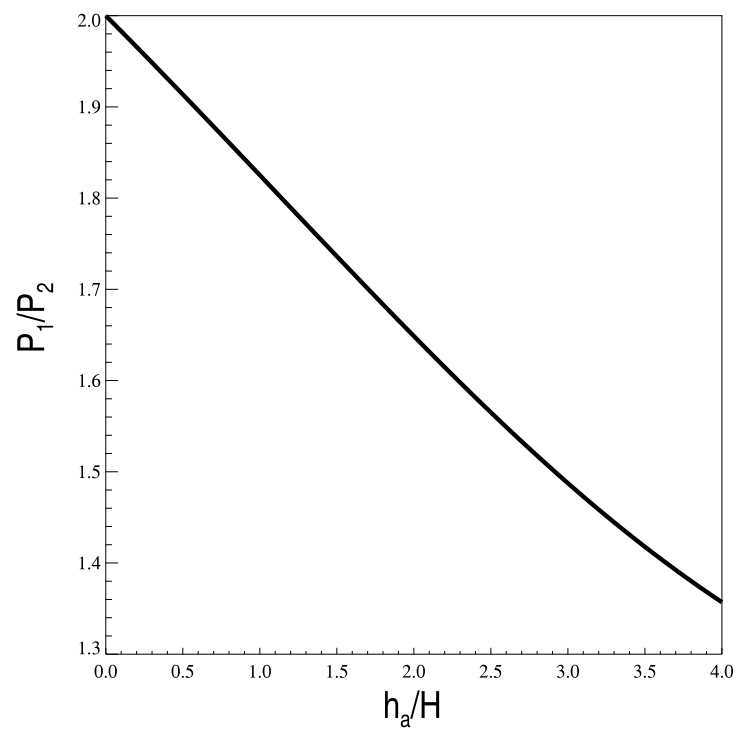

Fig. 2. Shown is the dependence of the period ratio on the loop stratification for a semi-circular loop.

period ratio, which is the ratio of the fundamental mode to the higher harmonics (or overtones). We can also find the frequency of the higher harmonics for the torsional Alfvén waves from Eq. (17). In Fig. 2 we show the period ratio of $P_{1} / P_{2}$, i.e. the ratio of the periods of the fundamental mode and the first overtone. The ratio of the periods can be seen to decrease from the canonical value, which is 2 for a homogenous loop, as the stratification increases. Comparing this figure to calculations of the period ratio for the fast kink mode (see, e.g. Andries et al. 2009; and Ruderman \& Erdélyi 2009, for reviews), we find that they are identical, i.e. stratification affects the frequency of the fast kink and Alfvén waves in exactly the same way. This may not be surprising due to the similar forms of Eq. (17) and the governing equation for fast kink modes in an inhomogeneous plasma (see, e.g., Dymova \& Ruderman 2005, 2006a; Morton \& Ruderman 2011). If we change to the dimensionless variables

$\Omega=\omega L / v_{\mathrm{Af}}, \quad \zeta=z / L$,

then Eq. (17) becomes

$\frac{\partial^{2} \xi_{\varphi}}{\partial \zeta^{2}}+\Omega^{2} \exp \left(-\frac{h_{\mathrm{a}}}{H} \cos \frac{\pi \zeta}{2}\right) \xi_{\varphi}=0$.

It is shown in Morton \& Ruderman (2011) that the governing equation for the fast kink mode also has the same form. This means that the eigenvalues, $\Omega$, are the same for both equations, hence independent of the phase speed. However, the observable frequencies, $\omega$, are different by a constant scaling factor. When the period ratio is taken, the scaling factors cancel and so we find the same trend in period ratio for both fast kink and Alfvén oscillations. The same reasoning also applies to changes in frequency/period when they are normalised with respect to the scaling factor.

\subsection{Spatio-seismology}

It has also been suggested and shown in Erdélyi \& Verth (2007) and Verth et al. (2007) that the amplitude profile of the first overtone of the fast kink oscillation can be exploited for magnetoseismological applications. This is an important and promising new magneto-seismological tool that should be exploited

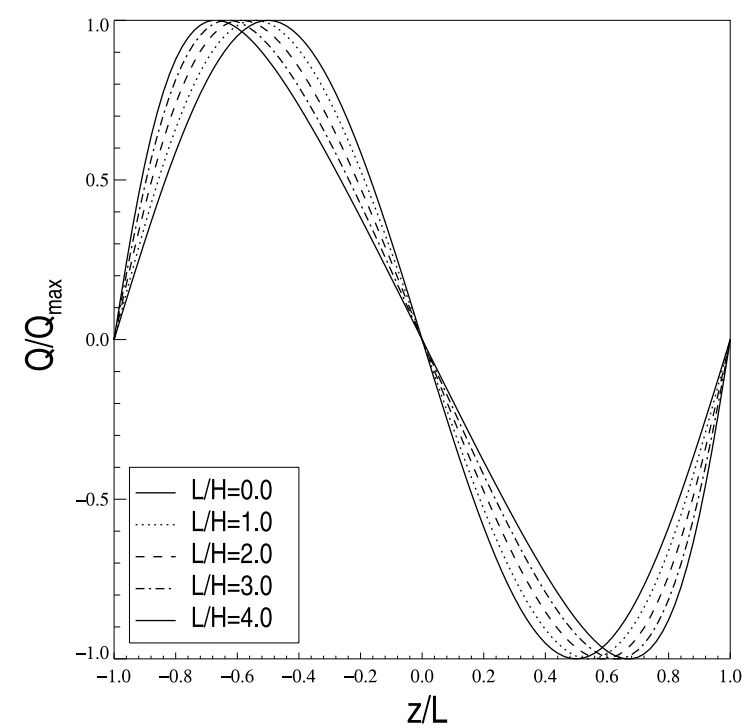

Fig. 3. Shown is the amplitude profile for the first overtone for different values of loop stratification. The values are normalised with respect to the maximum value of amplitude at each value of scale height.

by observations. It is known that the eigenfunctions (e.g. velocity, displacement, amplitudes) are more sensitive to background changes than frequencies. The current drawback is that instruments designed for coronal observations are more developed in their temporal resolution than their spatial. However, recent development in spectral imaging may considerably change the situation giving impetus to spatial seismology. Verth et al. (2007) demonstrated that the anti-nodes of the first overtone profile were subject to a shift in their spatial position due to stratification. As with the period ratio, here we expect to find a similar behaviour in the anti-node shifts as was found for the kink modes. The anti-node shifts are shown in Fig. 3 for a range of typical values of the ratio of loop length to scale height, where we have replaced loop height with loop length in Eq. (17) using $h_{\mathrm{a}}=2 L / \pi$. Comparing Fig. 3 to Fig. 2 in Verth et al. (2007) it can be seen that the shifts in the anti-nodes are comparable. Verth et al. (2007) also derived an analytic approximation for the anti-node shift, i.e.

$\frac{\triangle z_{\mathrm{AN}}}{L} \approx 0.025 \frac{L}{H}$,

where $\Delta z_{\mathrm{AN}}$ is the antinode shift. For a typical loop length of $120 \mathrm{Mm}$ and an average estimate of scale height, $H \sim 40 \mathrm{Mm}$, then we obtain the shift in the anti-node of $9 \mathrm{Mm}$. Shifts on these length scales correspond to almost a 2 pixel shift with resolution available onboard SDO/AIA (Tarbell \& AIA Team 2011).

Note that the spatio-seismology is a more sensitive method than the temporal seismology. The reason is that, if we change the shape of the loop while keeping the same loop length and the apex height, then the eigenfrequencies would change only slightly while the positions of nodes of the first overtone would change dramatically. As a result, to implement the spatioseismology, we need to know the precise shape of the loop, which is unavailable at present. Hopefully, we will be able to implement the spatio-seismology when future space missions similar to STEREO will be launched, but with much better spatial and temporal resolutions. 


\section{Alfvén waves in dynamic plasmas}

We now consider how dynamics in coronal loops will affect the properties of torsional Alfvén waves. First we investigate how steady flows will influence the oscillations. Then we will study how dynamic flows related to the mass loss in the loops, influence standing torsional Alfvén oscillations. The mass loss in the loops is likely to be caused by radiative cooling of the loops.

\subsection{Steady flows}

Consider a loop with the constant density and flow, $\rho=$ const. and $U=$ const. This uni-directional flow could essentially be a siphon flow along the loop between footpoints. Then, taking $\xi \sim \exp (-\mathrm{i} \omega t)$, we reduce Eq. (12) to

$\left(v_{\mathrm{A}}^{2}-U^{2}\right) \frac{\partial^{2} \xi_{\varphi}}{\partial z^{2}}+2 U \mathrm{i} \omega \frac{\partial \xi_{\varphi}}{\partial z}+\omega^{2} \xi_{\varphi}=0$.

The general solution to this equation is

$\xi_{\varphi}(z)=C_{1} \exp \left(\mathrm{i} k_{+} z\right)+C_{2} \exp \left(-\mathrm{i} k_{-} z\right)$

where $C_{1}$ and $C_{2}$ are constants and the wavenumbers $k_{+}$and $k_{-}$ are given by

$k_{ \pm}=\frac{\omega}{v_{\mathrm{A}} \pm U}$.

The wavenumbers here correspond to the forward and backward propagating waves. To find the solution corresponding to standing waves we have to solve a system of two linear homogenous algebraic equations for $C_{1}$ and $C_{2}$ obtained by substituting Eq. (23) into the boundary conditions (14),

$\left(\begin{array}{cc}\exp \left(\mathrm{i} k_{+} L\right) & \exp \left(\mathrm{i} k_{-} L\right) \\ \exp \left(-\mathrm{i} k_{+} L\right) & \exp \left(-\mathrm{i} k_{-} L\right)\end{array}\right)\left(\begin{array}{l}C_{1} \\ C_{2}\end{array}\right)=0$.

This system of equations only has non-trivial solutions when its determinant is zero. This condition gives the dispersion equation

$\exp \left(\frac{4 \mathrm{i} L \omega v_{\mathrm{A}}}{v_{\mathrm{A}}^{2}-U^{2}}\right)=1$.

The non-negative solutions to this equation are

$\omega_{n}=\frac{n \pi}{2 L} v_{\mathrm{A}}\left|1-\frac{U^{2}}{v_{\mathrm{A}}^{2}}\right|$,

where $n=1$ corresponds to the fundamental mode and $n>1$ to the overtones. Here the standing mode is now composed of forward and backward propagating waves with different wave numbers. We can see from Eq. (26) that the flow causes a change of the frequency of standing mode of the order $U^{2} / v_{\mathrm{A}}^{2}$. For typical estimates of Alfvén and flow speeds in the corona $U^{2} / v_{\mathrm{A}}^{2} \sim 0.01$, hence the change in frequency due to the flow is very small.

\subsection{Non-steady flows}

Now, assume that the coronal loop is subject to a radiative cooling process which causes a decrease in the loops density over time. The density profile takes the form of that given by Eq. (7). If a solution were sought to the profile of the flow then one would find that the flow was dependent upon distance along the loop and time, i.e. the flow would be non-steady. The assumption is made that the cooling process is occurring on a much slower timescale than the period of the Alfvén modes, i.e. $\tau_{\text {cool }} \gg P_{\mathrm{A}}$. We denote the ratio of these quantities as $\delta=P_{\mathrm{A}} / \tau_{\text {cool }}$, where $\delta \ll 1$. We can estimate the period of a torsional Alfvén wave as $P_{\mathrm{A}} \sim 2 L / v_{\mathrm{Ah}}$, where $v_{\mathrm{Ah}}$ is the characteristic Alfvén speed. Then we find that

$$
B \sim \delta^{-1} \sqrt{\mu_{0} \rho} \frac{L}{\tau_{\text {cool }}}
$$

This estimate inspires us to introduce a scaled magnetic field $\tilde{B}=\delta B$. We can now use the WKB approximation (see, e.g., Bender \& Orszag 1978) to obtain the approximate solutions to Eq. (12). In accordance with this method we look for the solution in the form

$\xi_{\varphi}=Q(z, t) \exp \left(\frac{\mathrm{i}}{\delta} \Theta(t)\right)$.

The amplitude function $Q$ is expanded in the power series with respect to $\delta$,

$Q(z, t)=Q_{0}+\delta Q_{1}+\ldots$

Substituting Eqs. (27) and (28) in Eq. (12) and collecting terms of order $\delta^{-2}$ (i.e. the lowest order) we obtain

$\frac{\partial^{2} Q_{0}}{\partial z^{2}}+\frac{\tilde{\omega}^{2}}{\tilde{v}_{\mathrm{A}}^{2}} Q_{0}=0$,

where $\tilde{v}_{\mathrm{A}}=\tilde{B} / \sqrt{\mu_{0} \rho}$ is the scaled Alfvén speed and

$$
\tilde{\omega}=\frac{\partial \Theta(t)}{\partial t} .
$$

It follows from Eq. (14) that $Q_{0}$ satisfies the boundary conditions

$Q_{0}=0 \quad$ at $\quad z= \pm L$.

Equations (29) and (30) define a boundary value problem of Sturm-Liouville type which determines the instantaneous frequency of the torsional standing Alfvén mode. This equation is the time-dependent version of Eq. (16).

Collecting terms of order $\delta^{-1}$ we obtain the next order approximation,

$\frac{\partial^{2} Q_{1}}{\partial z^{2}}+\frac{\tilde{\omega}^{2}}{\tilde{v}_{\mathrm{A}}^{2}} Q_{1}=-\frac{2 \mathrm{i} \tilde{\omega}}{\tilde{v}_{\mathrm{A}}^{2}}\left(\frac{\partial Q_{0}}{\partial t}+U \frac{\partial Q_{0}}{\partial z}+\frac{1}{2 \tilde{\omega}} \frac{\partial \tilde{\omega}}{\partial t} Q_{0}\right)$.

Again, the boundary conditions

$Q_{1}=0 \quad$ at $\quad z= \pm L$,

follow from Eq. (14). Equation (31) only has a solution if the right hand side satisfies a compatibility condition (Bender \& Orszag 1978). We can obtain this condition by multiplying (31) by $Q_{0}$, integrating with respect to $z$ and using boundary conditions (30). The compatibility condition is then given after some algebra by

$\omega \int_{-L}^{L} \frac{Q_{0}^{2}}{v_{\mathrm{A}}^{2}} \mathrm{~d} z=$ const.

where we have used Eq. (3) to eliminate $U$. In Eq. (33) $\omega$ and $v_{\mathrm{A}}$ are given by

$\omega=\delta^{-1} \tilde{\omega}, \quad v_{\mathrm{A}}=\delta^{-1} \tilde{v}_{\mathrm{A}}$.

Equation (33) determines how $Q_{0}$ evolves with time. It is similar to the corresponding equation derived by Ruderman (2011) for kink oscillations of loops with the slowly varying density. Equations of this type are called adiabatic invariants in the theory of systems with slowly varying parameters. 


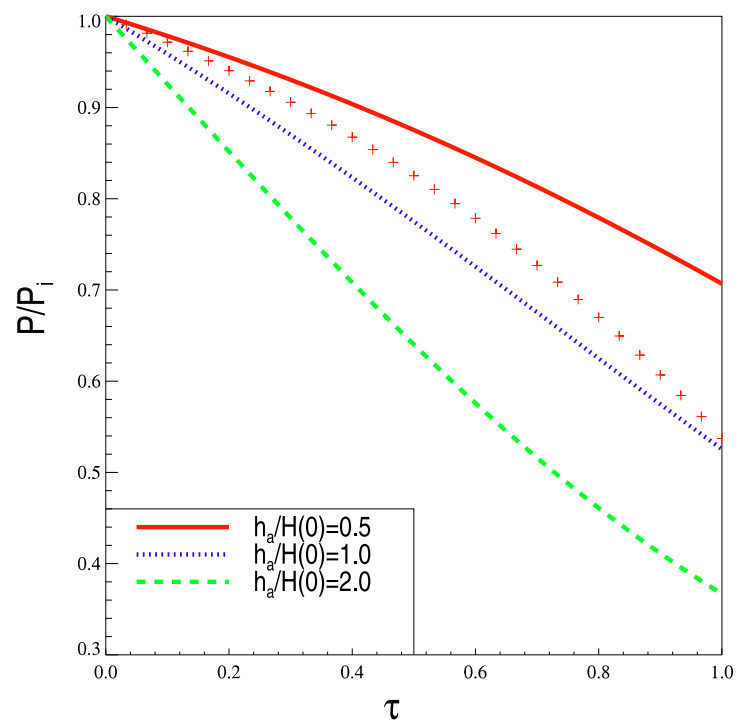

Fig. 4. Shown is the change in the period for fundamental Alfvén mode as a function of scaled time for a cooling coronal loop with different initial temperatures. The values are normalised with respect to the value of period at $\tau=0\left(P_{i}\right)$. The crosses are the approximate analytic solution for $h_{\mathrm{a}} / H(0)=0.5$.

\subsection{Cooling coronal loops}

We now investigate how the standing torsional Alfvén wave behaves in a cooling coronal loop. It is convenient to introduce the dimensionless time $\tau=t / t_{\text {cool }}$ and the ratio of the loop apex height to the atmospheric scale height at the initial moment of time $\kappa=h_{\mathrm{a}} / H(0)$. Then, using Eqs. (1) and (7), we reduce Eqs. (29) and (33) to

$\frac{\partial^{2} Q_{0}}{\partial \zeta^{2}}+\Omega^{2} \exp \left(-\kappa \mathrm{e}^{\tau} \cos \frac{\pi \zeta}{2}\right) Q_{0}=0$

$\Omega \int_{-1}^{1} Q_{0}^{2} \exp \left(-\kappa \mathrm{e}^{\tau} \cos \frac{\pi \zeta}{2}\right) \mathrm{d} \zeta=$ const.

where $\Omega$ and $\zeta$ are given by Eq. (19). The solution to Eq. (29) has to satisfy the boundary conditions

$Q_{0}=0 \quad$ at $\quad \zeta= \pm 1$.

Equation (35) was solved numerically using the shooting method. The results of these calculations are displayed in Figs. 4-6.

Figure 4 shows that the period of the standing torsional Alfvén waves decreases as the loop cools. Recall that the values of characteristic cooling time must be greater than the period of the Alfvén wave for the WKB theory to be applicable. We can find an estimate for the period in the corona where the typical value of Alfvén speed is $1 \mathrm{Mm} \mathrm{s}^{-1}$ and the typical loop lengths are $60-120 \mathrm{Mm}$. Using these numbers we obtain that the typical periods are $P=120-240 \mathrm{~s}$. We do not know how long Alfvén waves will actually exist before being damped or dissipated, so we plot for the typical lifetime of a transversal loop oscillation $(t \sim 1500 \mathrm{~s})$ as an indicative estimate. This, however, is not a statement or prediction on Alfvén wave lifetimes!

We can derive an expression for the period of the fundamental mode when $\kappa$ is small to demonstrate how an approximate solution looks and the role of $h_{\mathrm{a}} / H(t)$ in determining the period

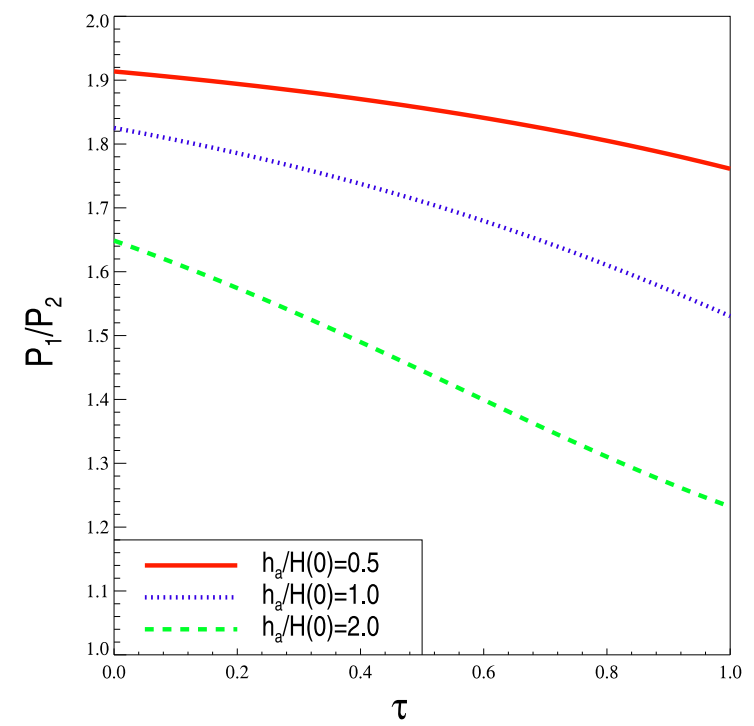

Fig. 5. Shown is the change in the period ratio of the fundamental mode to the first overtone as a function of scaled time for a cooling coronal loop for different values of $h_{\mathrm{a}} / H(0)$.

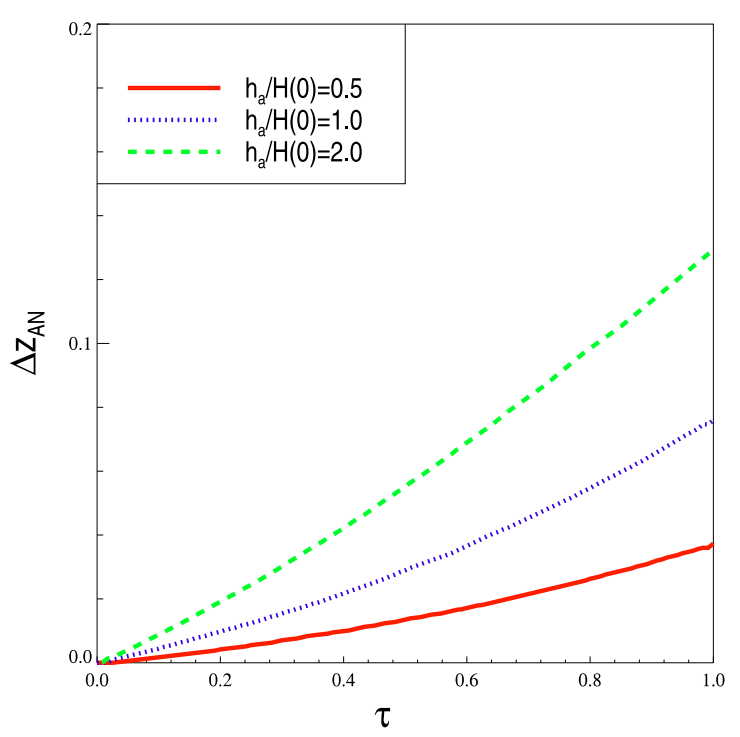

Fig. 6. Shown is the shift in the anti-node of the first overtone as a function of time for a cooling coronal loop for different values of $h_{\mathrm{a}} / H(0)$. The values calculated as the shift from the position at $\tau=0$.

of the oscillation. For this we expand the exponent in Eq. (35) in the power series and keep only the first two terms. As a result we obtain the approximate equation

$\frac{\partial^{2} Q_{0}}{\partial \zeta^{2}}+\Omega^{2} Q_{0}=\kappa \Omega^{2} Q_{0} \mathrm{e}^{\tau} \cos \frac{\pi \zeta}{2}$.

We are looking for the solution to this equation satisfying the boundary conditions (37) in the form of expansions

$Q_{0}=Q_{0}^{(0)}+\kappa Q_{0}^{(1)}+\ldots, \quad \Omega=\Omega^{(0)}+\kappa \Omega^{(1)}+\ldots$

Substituting these expansions in Eqs. (38) and (37), and collecting terms of the lowest order we obtain

$\frac{\partial^{2} Q_{0}^{(0)}}{\partial \zeta^{2}}+\Omega^{(0)^{2}} Q_{0}^{(0)}=0, \quad Q_{0}^{(0)}=0$ at $\zeta= \pm 1$. 
The solution corresponding to the fundamental mode is

$\Omega^{(0)}=\frac{\pi}{2}, \quad Q_{0}^{(0)}=\cos \frac{\pi \zeta}{2}$.

Proceeding to the next order approximation we collect terms proportional to $\kappa$ in Eqs. (38) and (37) and use Eq. (41) to obtain

$\frac{\partial^{2} Q_{0}^{(1)}}{\partial \zeta^{2}}+\Omega^{(0)^{2}} Q_{0}^{(1)}=-\pi \Omega^{(1)} \cos \frac{\pi \zeta}{2}+\frac{\pi^{2}}{4} \mathrm{e}^{\tau} \cos ^{2} \frac{\pi \zeta}{2}$,

$Q_{0}^{(1)}=0 \quad$ at $\quad \zeta= \pm 1$.

This boundary-value problem has a solution only when the righthand side of Eq. (42) satisfies the compatibility condition. To obtain this condition we multiply Eq. (42) by $\cos (\pi \zeta / 2)$, integrate with respect to $\zeta$ from -1 to 1 , and use integration by parts and Eq. (43). As a result we obtain

$\int_{-1}^{1}\left(-\pi \Omega^{(1)} \cos \frac{\pi \zeta}{2}+\frac{\pi^{2}}{4} \mathrm{e}^{\tau} \cos ^{2} \frac{\pi \zeta}{2}\right) \cos \frac{\pi \zeta}{2} \mathrm{~d} \zeta=0$.

Calculating this integral we eventually arrive at

$\Omega^{(1)}=\frac{2 \mathrm{e}^{\tau}}{3}$.

Then, using Eqs. (19) and (39) and the relation between the period and frequency, $P=2 \pi / \omega$, we obtain the approximate expression for the period of the fundamental mode,

$P(t)=\frac{4 L}{V_{\mathrm{Af}}}\left(1-\frac{4 h_{\mathrm{a}}}{3 \pi H(t)}\right)$.

Note that the condition $\kappa=h_{\mathrm{a}} / H(0) \ll 1$ is not sufficient to guarantee that this approximate expression for $P$ is valid. We need to impose a stronger restriction $h_{\mathrm{a}} / H(t)=\kappa \mathrm{e}^{\tau} \ll 1$. The relation $\kappa e^{\tau} \ll 1$ is satisfied reasonably well for typical values of $H \sim 47-70 \mathrm{Mm}$ for the temperature range $T \sim 1-1.5 \mathrm{MK}$ and for loops of heights $15-23 \mathrm{Mm}$. We plot Eq. (46) in Fig. 4 for comparison between the analytical approximation and the numerical solution of Eq. (35) for $h_{\mathrm{a}} / H(0)=0.5$.

In Fig. 5 we plot the period ratio as a function of the scaled time for a cooling loop. It can be seen that the period ratio does not begin at 2 for each curve. This is because the loop is already stratified at the initial moment of time. Figure 5 clearly shows that the loop is becoming more stratified as it cools. This is also seen in Fig. 6 where we show the anti-node shift as function of scaled time. The shift from its original position is increasing, which, comparing to the results in Fig. 3, shows that stratification is increasing as the loop cools. Estimates of the size of the expected anti-node shifts for typical coronal loops are given in Sect. 3.2.

Now, we turn to Eq. (33) to study the evolution of the amplitude of the fundamental torsional Alfvén mode. We write the amplitude function as $Q_{0}=S(\tau) Z(\zeta, \tau)$, where $Z(\zeta, \tau)$ is the solution of the boundary-value problem defined by Eqs. (35) and (37) satisfying the condition $Z(0, \tau)=1$. Substituting this expression in Eq. (36) we obtain

$\Omega S^{2}(\tau) \int_{-1}^{1} Z^{2}(\tau, \zeta) \exp \left(\kappa \mathrm{e}^{\tau} \cos \frac{\pi \zeta}{2}\right) \mathrm{d} \zeta=$ const.

Obviously $S(\tau)$ is equal to the oscillation amplitude at the apex point. We solved Eq. (47) numerically and plotted the amplitude

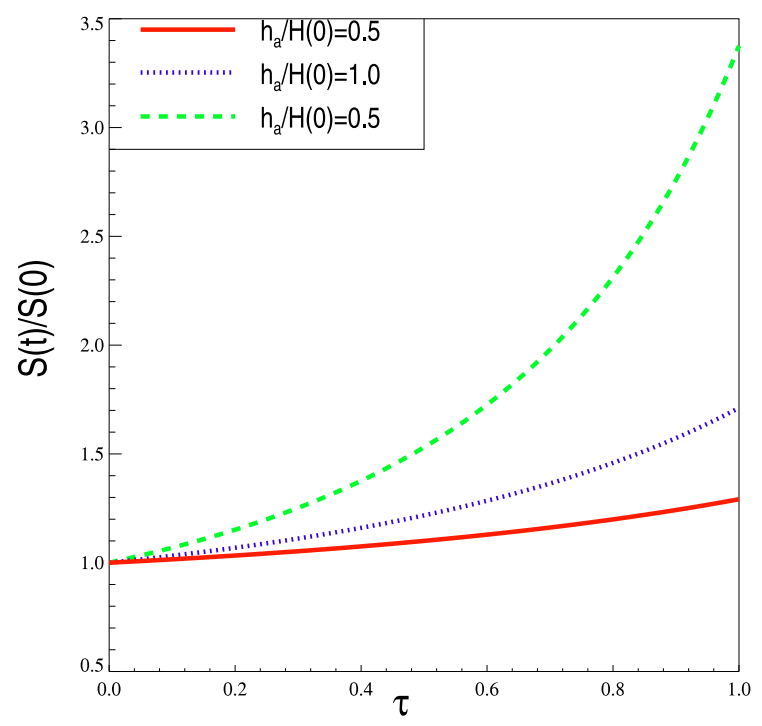

Fig. 7. Shown is the change in amplitude of the fundamental mode as a function of time for a cooling coronal loop with different values of $h_{\mathrm{a}} / H(0)$. The values are normalised with respect to the value of amplitude at $\tau=0$.

as a function of dimensionless time in Fig. 7. It is clearly seen that, as the density decreases (as the loop cools), there is an increase in the amplitude of the mode. This is the behaviour similar to that obtained by Ruderman (2011) for fast kink oscillations in a cooling loop. However, the amplitude of the Alfvén wave is found to increase more rapidly than the amplitude of the fast kink mode. If both the plasma inside and outside the loop was assumed to be cooling then we would obtain the same results for the fast kink mode and the torsional Alfvén mode.

\section{Discussion and conclusions}

This paper is the next step in developing the fundamental theory of MHD waves in dynamic (i.e. time-dependent background) plasmas (see also Morton \& Erdélyi 2009b; Morton et al. 2010; Erdélyi et al. 2011; Ruderman 2011). Here we obtain and solve the governing equations for standing torsional Alfvén waves in static, stratified plasmas and in dynamic stratified plasmas. We also demonstrate the possibility of the use of standing torsional Alfvén waves as magneto-seismological tools. It is found that the standing torsional Alfvén waves display similar behaviour to that found previously for fast kink oscillations, i.e. as the loop becomes increasingly stratified: $(i)$ the period ratio of the fundamental mode and the first overtone decreases from the canonical value 2 (Andries et al. 2009; Ruderman \& Erdélyi 2009); and (ii) the amplitude profile of the first overtone experiences a spatial shift of its anti-nodes (Verth et al. 2007).

This naturally leads to the suggestion that Alfvén modes will also provide both temporal and spatial magneto-seismological tools for obtaining estimates of the density scale height. However, there are some complications associated with using the Alfvén wave as a magneto-seismological tool. Coronal loops are thought to have a non-constant radial density which leads to the idea of defining individual magnetic surfaces within the loop based on the values of Alfvén speeds at each surface. It has been shown (e.g. Heyvaerts \& Priest 1983; Ruderman 1999) that the driving of torsional Alfvén modes by a random footpoint driver leads to the excitement of non-collective oscillatory 
behavior of different magnetic surfaces in the loop. This means that unless we can identify the magnetic surface on which any measured torsional oscillation occurs then the use of the period ratio will be limited at present, i.e. the scenario may arise that we observe the fundamental mode of one surface and the first overtone of another surface which would lead to inaccurate estimates if using the period ratio. Only when higher resolution satellites (e.g. with an estimated spatial resolution $\lesssim 50 \mathrm{~km}$ ) are developed will the period ratio become a useful tool. The more appropriate tools for obtaining the estimates of density scale height may be the anti-node shifts which only require measurements along one magnetic surface. However, as we have already noted, to use the anti-node shift as a seismological tool we need to know the loop shape accurately.

Another complication related to using Alfvén waves as a seismological tool is phase mixing. Assume that we are able to observe oscillations in the vicinity of a particular magnetic surface in a coronal loop. Let the spatial resolution be $\Delta r$ and the loop radius is $R, \Delta r \ll R$. Assume also that the characteristic spatial scale of the density and, consequently, the Alfvén speed in the radial direction is $R$. Finally, assume that, at the initial moment of time, quasi-coherent torsional Alfvén waves are excited in the vicinity of a particular magnetic surface with the size in the radial direction of the order of $\Delta r$. Then, due to the phase mixing, the phase difference of oscillations of the neighbouring magnetic surfaces will be of the order of $t \Delta r / R P$, where $P$ is the wave period. After the time of the order of $P R / \Delta r$ the motion will become completely incoherent and we will not be able to observe a standing torsional mode. We will observe the coherent motion only for the time interval determined by the condition $t \ll P R / \Delta r$. Hence, it is crucial to have $\Delta r \ll R$. If we take, for example, $\Delta r=50 \mathrm{~km}$ and $R=1 \mathrm{Mm}$, then we can hope to observe the coherent torsional oscillation only for about 3 to 4 periods.

For the dynamic plasma, as the loop cools and losses mass, the standing torsional Alfvén wave experiences an increase in frequency and amplitude. The rate at which the wave properties change is dependent upon the characteristic cooling timescale of the loop, the apex point height and the initial scale height. The period ratio and anti-node shift also show time-dependent behaviour due to the increasing loop stratification as the loop cools. The anti-node shifts should be within the observational limits of SDO. This is again similar to the behaviour of the fast kink modes in a cooling coronal loop (Morton \& Erdélyi 2009b; Ruderman 2011).

Using the time-dependent properties of the torsional Alfvén wave, i.e. the period change, it should be possible to obtain estimates for the cooling timescales in coronal loops. This is useful to test whether the values obtained from filter based techniques (e.g., Aschwanden \& Terradas 2008) are accurate. Further, it may be possible to exploit the rate of change of period to estimate the initial scale height and, hence, the range of scale heights of loop if both the cooling timescale and loop height are known, i.e. as different initial temperatures give different rates of change of period.

A further, important conclusion can also be drawn here. We show that the fast kink oscillations and torsional Alfvén waves obey the same dimensionless governing equations in static plasmas. Hence, the different waves react in exactly the same manner to the introduction of stratification. In fact, interestingly, for longitudinal oscillations the same dimensionless Sturm-Liouville equation is obtained in static plasmas, as found recently by Luna-Cardozo et al. (2011). This situation changes when dynamic behaviour is introduced and the governing equations for the fast kink and Alfvén wave differ.

Although there are no observations of the coronal torsional Alfvén waves to date, they are undoubtedly present in the solar atmosphere as evidenced by Jess et al. (2009) below the transition region and predicted numerically by Fedun et al. (2011b). This makes it very useful to investigate its behaviour in typical coronal situations so that it may be exploited accurately once observed.

We should also add that the study of cooling is far from trivial. The density profile obtained for this system is an approximate solution to the hydrodynamic equations governing the thermodynamic behaviour of the loop. Mass loss from the loop could be much lower than predicted here if there is also some heating of the loop plasma (causing chromospheric evaporation). The results here demonstrate the expected behaviour of waves in a cooling loop but further (probably numerical) calculations, using the full hydrodynamic equations to determine the background profile, need to be carried out.

Acknowledgements. R.E. acknowledges M. Kéray for patient encouragement. The authors are also grateful to NSF, Hungary (OTKA, Ref. No. K83133) and the Science and Technology Facilities Council (STFC), UK for the financial support they received.

\section{References}

Andries, J., Arregui, I., \& Goossens, M. 2005, ApJ, 624, L57

Andries, J., van Doorsselaere, T., Roberts, B., et al. 2009, Space Sci. Rev., 149, 3

Aschwanden, M. J., \& Terradas, J. 2008, ApJ, 686, L127

Aschwanden, M. J., \& Tsiklauri, D. 2009, ApJS, 185, 171

Aschwanden, M. J., Fletcher, L., Schrijver, C. J., \& Alexander, D. 1999, ApJ, 520,880

Banerjee, D., Erdélyi, R., Oliver, R., \& O’Shea, E. 2007, Sol. Phys., 246, 3

Bender, C. M., \& Orszag, S. A. 1978, Advanced Mathematical Methods for Scientists and Engineers (New York: McGraw-Hill)

Bonet, J. A., Márquez, I., Sánchez Almeida, J., Cabello, I., \& Domingo, V. 2008, ApJ, 687, L131

Coddington, E. A., \& Levinson, N. 1955, Theory of Ordinary Differential Equations (New York: McGraw-Hill)

De Moortel, I. 2009, Space Sci. Rev., 149, 65

Del Zanna, G. 2008, A\&A, 481, L49

Dymova, M. V., \& Ruderman, M. S. 2005, Sol. Phys., 229, 79

Dymova, M. V., \& Ruderman, M. S. 2006a, A\&A, 457, 1059

Dymova, M. V., \& Ruderman, M. S. 2006b, A\&A, 459, 241

Edwin, P. M., \& Roberts, B. 1983, Sol. Phys., 88, 179

Erdelyi, R. 1998, Sol. Phys., 180, 213

Erdélyi, R., \& Fedun, V. 2010, Sol. Phys., 263, 63

Erdelyi, R., \& Goossens, M. 1995, A\&A, 294, 575

Erdélyi, R., \& Morton, R. J. 2009, A\&A, 494, 295

Erdélyi, R., \& Taroyan, Y. 2008, A\&A, 489, L49

Erdélyi, R., \& Verth, G. 2007, A\&A, 462, 743

Erdélyi, R., Goossens, M., \& Ruderman, M. S. 1995, Sol. Phys., 161, 123

Erdélyi, R., Doyle, J. G., Perez, M. E., \& Wilhelm, K. 1998, A\&A, 337, 287

Erdélyi, R., Al-Ghafri, K. S., \& Morton, R. J. 2011, Sol. Phys., 272, 73

Fedun, V., Shelyag, S., \& Erdélyi, R. 2011a, ApJ, 727, 17

Fedun, V., Shelyag, S., Verth, G., Mathioudakis, M., \& Erdélyi, R. 2011b, Ann. Geophys., submitted

Goossens, M., Hollweg, J. V., \& Sakurai, T. 1992, Sol. Phys., 138, 233

Goossens, M., Andries, J., \& Aschwanden, M. J. 2002, A\&A, 394, L39

Heyvaerts, J., \& Priest, E. R. 1983, A\&A, 117, 220

Jess, D. B., Mathioudakis, M., Erdélyi, R., et al. 2009, Science, 323, 1582

Klimchuk, J. A., Karpen, J. T., \& Antiochos, S. K. 2010, ApJ, 714, 1239

Luna-Cardozo, M., Verth, G., \& Erdélyi, R. 2011, ApJ, submitted

Morton, R., \& Erdélyi, R. 2009a, A\&A, 605, 493

Morton, R. J., \& Erdélyi, R. 2009b, ApJ, 707, 750

Morton, R. J., \& Ruderman, M. S. 2011, A\&A, 527, A53

Morton, R. J., Hood, A. W., \& Erdélyi, R. 2010, A\&A, 512, A23

Morton, R. J., Erdélyi, R., Jess, D. B., \& Mathioudakis, M. 2011, ApJ, 729, L18 


\section{R. J. Morton et al.: Torsional Alfvén waves}

Ofman, L., \& Wang, T. J. 2008, A\&A, 482, L9

Ruderman, M. S. 1999, ApJ, 521, 851

Ruderman, M. S. 2003, A\&A, 409, 287

Ruderman, M. S. 2009, A\&A, 506, 885

Ruderman, M. S. 2010, Sol. Phys., 267, 377

Ruderman, M. S. 2011, Sol. Phys., 271, 41

Ruderman, M. S., \& Erdélyi, R. 2009, Space Sci. Rev., 149, 199

Ruderman, M. S., \& Roberts, B. 2002, ApJ, 577, 475

Ruderman, M. S., Verth, G., \& Erdélyi, R. 2008, ApJ, 686, 694

Schrijver, C. J., Title, A. M., Berger, T. E., et al. 1999, Sol. Phys., 187, 261

Shelyag, S., Keys, P., Mathioudakis, M., \& Keenan, F. P. 2011, A\&A, 526, A5

Tarbell, T. D., \& AIA Team. 2011, in Am. Astron. Soc. Meeting Abstracts, 217, 155.09

Taroyan, Y., \& Erdélyi, R. 2008, Sol. Phys., 251, 523

Taroyan, Y., \& Erdélyi, R. 2009, Space Sci. Rev., 24
Terradas, J., Oliver, R., \& Ballester, J. L. 2006, ApJ, 642, 533

Terradas, J., Goossens, M., \& Ballai, I. 2010, A\&A, 515, A46

Tomczyk, S., McIntosh, S. W., Keil, S. L., et al. 2007, Science, 317, 1192

Tripathi, D., Mason, H. E., Dwivedi, B. N., del Zanna, G., \& Young, P. R. 2009 ApJ, 694, 1256

van Doorsselaere, T., Verwichte, E., \& Terradas, J. 2009, Space Sci. Rev., 149, 299

Verth, G., \& Erdélyi, R. 2008, A\&A, 486, 1015

Verth, G., van Doorsselaere, T., Erdélyi, R., \& Goossens, M. 2007, A\&A, 475, 341

Verth, G., Erdélyi, R., \& Goossens, M. 2010, ApJ, 714, 1637

Wang, T. J., Solanki, S. K., Innes, D. E., Curdt, W., \& Marsch, E. 2003, A\&A, 402, L17

Zaqarashvili, T. V. 2003, A\&A, 399, L15

Zaqarashvili, T. V., \& Murawski, K. 2007, A\&A, 470, 353 